\title{
Effect of perioperative sodium bicarbonate on postoperative acute kidney injury in infective endocarditis patients
}

\section{Jin Sun Cho, Young Ran Kang, Sung Yeon Ham, Young Lan Kwak \\ Department of Anesthesiology and Pain Medicine, Yonsei University College of Medicine}

\section{Background}

- Acute kidney injury (AKI) following cardiac surgery is associated with inflammation, oxidative stress, and generation of oxygen free radicals.

- Patients with infective endocarditis (IE) have a high risk of AKI due to systemic inflammation and infection.

- Sodium bicarbonate has intrinsic natriuretic effects and ability to alkalize tubular fluid, increasing oxygen delivery and reducing oxidative stress by free radical formation.

-We investigated whether perioperative sodium bicarbonate infusion could attenuate AKI in IE patients undergoing valvular heart surgery

\section{Methods}

\section{Inclusion criteria}

- IE patients (> 20 years) undergoing elective valvular heart surgery with cardiopulmonary bypass

\section{Exclusion criteria}

- LV ejection fraction less than 30\%, congestive heart failure, renal insufficiency (eGFR $<15 \mathrm{ml} / \mathrm{min}$ per $1.73 \mathrm{~m}^{2}$ )

\section{Study groups}

- Control group: saline infusion

- Bicarbonate group: sodium bicarbonate was administered at a loading dose of $0.5 \mathrm{mmol} / \mathrm{kg}$ for $1 \mathrm{~h}$ commencing with anesthetic induction, followed by an infusion rate of 0.15 $\mathrm{mmol} / \mathrm{kg} / \mathrm{h}$ for $23 \mathrm{~h}$.

\section{Primary outcome:}

- the incidence of AKI during the first postoperative $48 \mathrm{~h}$

\section{Secondary outcomes}

- serum creatinine level and glomerular filtration rate during the postoperative 5 days, and major morbidity endpoints

\section{Results}

Table 1. Patient characteristics

\begin{tabular}{|l|c|c|c|}
\hline & $\begin{array}{c}\text { Control group } \\
(\mathbf{n}=33)\end{array}$ & $\begin{array}{c}\text { Bicarbonate group } \\
(\mathbf{n = 2 9 )}\end{array}$ & P-value \\
\hline Age (years) & $54.4 \pm 15.9$ & $53.8 \pm 14.6$ & 0.881 \\
\hline Sex (M:F) & $24: 9$ & $17: 12$ & 0.118 \\
\hline Body mass index $\left(\mathrm{kg} / \mathrm{m}^{2}\right)$ & $22.0 \pm 3.5$ & $22.0 \pm 3.3$ & 0.991 \\
\hline Hypertension & 7 & 10 & 0.307 \\
\hline Diabetes mellitus & 4 & 3 & 0.758 \\
\hline Congestive heart failure & 1 & 0 & 0.329 \\
\hline Chronic renal failure & 1 & 2 & 0.514 \\
\hline Cerebrovascular disease & 4 & 3 & 0.758 \\
\hline ACEi/ARB & $4 / 5$ & $6 / 4$ & $0.419 / 0.800$ \\
\hline 3-blockers & 6 & 7 & 0.653 \\
\hline calcium channel blockers & 2 & 4 & 0.344 \\
\hline diuretics & 10 & 13 & 0.317 \\
\hline Redo & 3 & 5 & 0.389 \\
\hline Complex heart surgery & 5 & 6 & 0.569 \\
\hline Preoperative ejection fraction $(\%)$ & $64.5 \pm 8.6$ & $66.6 \pm 7.1$ & 0.303 \\
\hline EuroSCORE & $5.5 \pm 3.5$ & $6.1 \pm 3.1$ & 0.487 \\
\hline Cleveland Clinic Score* & $1.5 \pm 1.0$ & $1.9 \pm 0.7$ & 0.097 \\
\hline Anesthesia time (min) & $273.4 \pm 78.0$ & $264.2 \pm 105.4$ & 0.703 \\
\hline Cardiopulmonary bypass time (min) & $123.0 \pm 53.0$ & $112.2 \pm 77.6$ & 0.529 \\
\hline & & & \\
\hline
\end{tabular}

Table 2. Incidence of acute kidney injury

\begin{tabular}{|l|c|c|c|}
\hline AKIN classification & $\begin{array}{c}\text { Control group } \\
(\mathbf{n}=33)\end{array}$ & $\begin{array}{c}\text { Bicarbonate group } \\
(\mathbf{n}=29)\end{array}$ & P-value \\
\hline None $\mathbf{( n )}$ & $26(79 \%)$ & $20(69 \%)$ & 0.459 \\
\hline Stage 1 (n) & $6(18 \%)$ & $5(17 \%)$ & 0.833 \\
\hline Stage 2 (n) & $1(3 \%)$ & $2(7 \%)$ & 0.514 \\
\hline Stage 3 (n) & 0 & $2(7 \%)$ & 0.137 \\
\hline Any category & $7(21 \%)$ & $9(31 \%)$ & 0.459 \\
\hline
\end{tabular}

Table 3. Serum creatinine and glomerular filtration rate

\begin{tabular}{|c|c|c|c|c|}
\hline Variables & Time points & $\begin{array}{l}\text { Control group } \\
(n=33)\end{array}$ & $\begin{array}{l}\text { Bicarbonate group } \\
(n=29)\end{array}$ & $\mathbf{P}_{\text {Group } \times \text { Time }}$ \\
\hline \multirow{6}{*}{$\begin{array}{l}\text { Serum creatinine } \\
(\mathrm{mg} / \mathrm{dl})\end{array}$} & Tо & $0.83 \pm 0.23$ & $0.85 \pm 0.24$ & \multirow{6}{*}{0.077} \\
\hline & T1 & $0.77 \pm 0.26^{*}$ & $0.73 \pm 0.23^{*}$ & \\
\hline & $\mathrm{T} 2$ & $0.95 \pm 0.32^{*}$ & $1.03 \pm 0.42^{*}$ & \\
\hline & T3 & $0.90 \pm 0.34$ & $1.19 \pm 0.70^{*}, \dagger$ & \\
\hline & T4 & $0.84 \pm 0.31$ & $1.15 \pm 0.75^{*}$ & \\
\hline & T5 & $0.80 \pm 0.26$ & $1.03 \pm 0.69$ & \\
\hline \multirow{6}{*}{$\begin{array}{l}\text { Glomerular } \\
\text { filtration rate } \\
(\mathrm{ml} / \mathrm{min} \text { per } 1.73 \\
\left.\mathrm{m}^{2}\right)\end{array}$} & TO & $92.2 \pm 32.4$ & $86.9 \pm 36.9$ & \multirow{6}{*}{0.083} \\
\hline & $\mathrm{T} 1$ & $103.6 \pm 40.9^{*}$ & $102.7 \pm 44.4^{*}$ & \\
\hline & T2 & $83.1 \pm 34.0^{*}$ & $78.7 \pm 40.8$ & \\
\hline & T3 & $90.1 \pm 39.3$ & $72.5 \pm 38.8^{*}$ & \\
\hline & T4 & $96.5 \pm 38.7$ & $77.3 \pm 40.3$ & \\
\hline & T5 & $99.5 \pm 34.2$ & $84.9 \pm 44.7$ & \\
\hline
\end{tabular}

Table 4. Postoperative outcomes

\begin{tabular}{|l|c|c|c|}
\hline & $\begin{array}{c}\text { Control group } \\
(\mathbf{n}=33)\end{array}$ & $\begin{array}{c}\text { Bicarbonate group } \\
(\mathbf{n}=\mathbf{2 9})\end{array}$ & P value \\
\hline Composite of morbidity endpoints & 8 & 11 & 0.369 \\
\hline permanent stroke & 3 & 0 & 0.073 \\
\hline hemostatic reoperation & 3 & 4 & 0.681 \\
\hline acute kidney injury & 4 & 9 & 0.102 \\
\hline ventilator care >48 $\mathbf{~}$ & 1 & 2 & 0.550 \\
\hline deep sternal wound infection & 0 & 1 & 0.312 \\
\hline in-hospital mortality & 1 & 0 & 0.312 \\
\hline Duration of ventilator care (hour) & $18[14,19]$ & $16[9,22]$ & 0.991 \\
\hline Duration of ICU stay (day) & $2[2,3]$ & $2[1,3]$ & 0.881 \\
\hline Duration of hospital stay (day) & $23[18,34]$ & $19[16,32]$ & 0.524 \\
\hline
\end{tabular}

\section{Conclusions}

Perioperative sodium bicarbonate administration did not demonstrate the favourable impact on renal function in IE patients undergoing cardiac surgery. 